SUPPORTING INFORMATION for IC060318d

Comparative Bonding Behaviour of Functional Cyclopentadienyl Ligands and BoronContaining Analogs in Heterometallic Complexes and Clusters.

Pierre Croizat, ${ }^{a}$ Nicolas Auvray, ${ }^{a}$ Pierre Braunstein, ${ }^{a}$ Richard Welter ${ }^{b}$

a Laboratoire de Chimie de Coordination, UMR 7177 CNRS, Université Louis Pasteur, 67070 Strasbourg Cédex, France.E-mail: braunst@chimie.u-strasbg.fr

b Laboratoire DECOMET, UMR 7177 CNRS, Université Louis Pasteur, 67070 Strasbourg Cédex, France.

Received

Contains Table S-1 for hydrogen bonding interactions and two Figures 
Table S-1. Hydrogen Bonding in the crystal structures

\section{Complex 10:}

Bifurcated $\mathrm{H}$ bond from $\mathrm{O} 1$ to $\mathrm{O} 1$ and $\mathrm{O} 2$

\section{Complex 11:}

\begin{tabular}{|c|c|c|c|c|c|c|}
\hline Nr Typ Res & Donor --- H....Acceptor & [ARU ] & $\mathrm{D}-\mathrm{H}$ & H...A & D...A I & - H...A \\
\hline 1 & C(14) --H(14) ..O(3) & {$[4565.01]$} & 0.95 & 2.53 & $3.376(9)$ & 149 \\
\hline 2 Intra 1 & $\mathrm{C}(15)--\mathrm{H}(15 \mathrm{~B}) . . \mathrm{O}(1)$ & ] & 0.99 & 2.49 & $3.241(8)$ & 132 \\
\hline 3 Intra 1 & C(17) --H(17B) ..O(2) & ] & 0.99 & 2.40 & $3.145(9)$ & 131 \\
\hline 1 & C(19) --H(19A) ..O(1) & [4554.01] & 0.99 & 2.46 & $3.326(9)$ & 146 \\
\hline
\end{tabular}

No Classic Hydrogen Bonds Found

Translation of ARU-code to Equivalent Position Code

\begin{tabular}{|c|}
\hline 4554. ] $=x, 1 / 2-y,-1 / 2+z$ \\
\hline 4565. ] =x,3/2-y,1/2+z \\
\hline
\end{tabular}

\section{Complex 12:}

$\begin{array}{lllllllr}\text { Nr Typ Res } & \text { Donor --- H....Acceptor [ ARU ] } & \text { D - H } & \text { H...A } & \text { D...A } & \text { D - H...A } \\ 1 & 1 * & \text { C14 --H14 ..O1 } & {[1455.01]} & 0.95 & 2.41 & 3.060(9) & 125\end{array}$

No Classic Hydrogen Bonds Found

Translation of ARU-code to Equivalent Position Code

[ 1455. ] $=-1+x, y, z$ 


\section{Complex 13:}

Nr Typ Res Donor --- H....Acceptor [ ARU ] D - H H...A D...A D - H...A

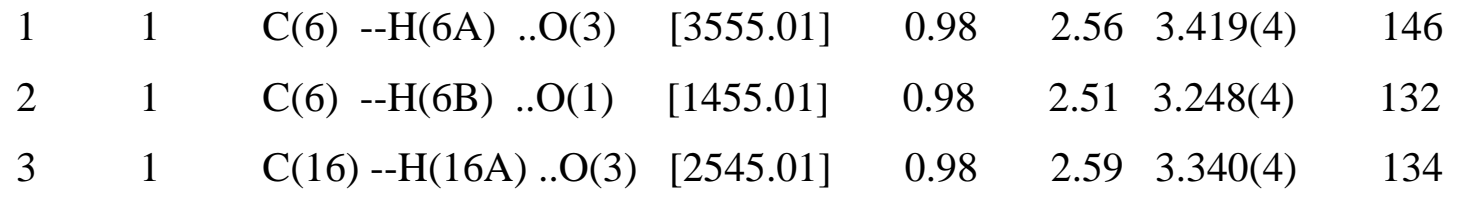

No Classic Hydrogen Bonds Found

Translation of ARU-code to Equivalent Position Code

\begin{tabular}{|c|}
\hline [ 3555. ] = -x,-y,-z \\
\hline [ 1455. ] $=-1+x, y, z$ \\
\hline 2545. $]=1 / 2-x,-1 / 2+y, 1 / 2-z$ \\
\hline
\end{tabular}

\section{Complex 15:}

\begin{tabular}{|c|c|c|c|c|c|c|}
\hline Nr Typ Res & Donor --- H....Accepto & r [ ARU ] & $\mathrm{D}-\mathrm{H}$ & H...A & D...A & D - H...A \\
\hline 1 & $\mathrm{C}(5)--\mathrm{H}(5)$ & [2667.01] & 0.95 & 2.40 & $3.328(5)$ & 165 \\
\hline 2 Intra 1 & C(10) --H(10C)..O(3) & ] & 0.98 & 2.52 & $3.320(6)$ & 139 \\
\hline 3 Intra 1 & $\mathrm{C}(11)--\mathrm{H}(11 \mathrm{~A}) . . \mathrm{O}(1)$ & {$[2677.01]$} & 0.99 & 2.55 & $3.272(5)$ & 130 \\
\hline Intra 1 & $\mathrm{C}(15)$--H(15A)..O(2) & 1 & 0.99 & 2.56 & $3.288(5)$ & 131 \\
\hline
\end{tabular}

No Classic Hydrogen Bonds Found

Translation of ARU-code to Equivalent Position Code

$\left[\begin{array}{l}2677 .] \\ {[2667 .]}\end{array}=1-x, 2-y, 2-z\right.$
$[-y, 2-z$ 


\section{Complex 16:}

\begin{tabular}{|c|c|c|c|c|c|c|}
\hline Nr Typ Res & Donor --- H....Accepto & r [ ARU ] & $\mathrm{D}-\mathrm{H}$ & H...A & D...A & - H...A \\
\hline 1 & $\mathrm{C}(6)--\mathrm{H}(6) . . \mathrm{O}(2)$ & [2657.01] & 0.95 & 2.45 & $3.305(16)$ & 150 \\
\hline 2 Intra 1 & $\mathrm{C}(10)--\mathrm{H}(10 \mathrm{~A}) . . \mathrm{O}(3)$ & ] & 0.98 & 2.53 & $3.348(17)$ & 140 \\
\hline 3 Intra 1 & (11) $--\mathrm{H}(11 \mathrm{~B}) . . \mathrm{O}(1)$ & {$[2667.01]$} & 0.99 & 2.47 & $3.160(14)$ & 126 \\
\hline Intra 1 & C(13) --H(13A)..O(2) & ] & 0.99 & 2.54 & $3.225(14)$ & 127 \\
\hline
\end{tabular}

No Classic Hydrogen Bonds Found

Translation of ARU-code to Equivalent Position Code

\begin{tabular}{|c|}
\hline [ 2667. ] = 1-x,1-y,2-z \\
\hline$[2657]=.1-x,-y, 2-z$ \\
\hline
\end{tabular}

\section{Complex 20:}

$\begin{array}{lllllll}\text { Nr Typ Res } & \text { Donor --- H....Acceptor [ ARU ] } & \text { D - H } & \text { H...A } & \text { D...A } & \text { D - H...A }\end{array}$

No Classic Hydrogen Bonds Found

Translation of ARU-code to Equivalent Position Code

[ 2675. ] = 1-x,2-y,-z 


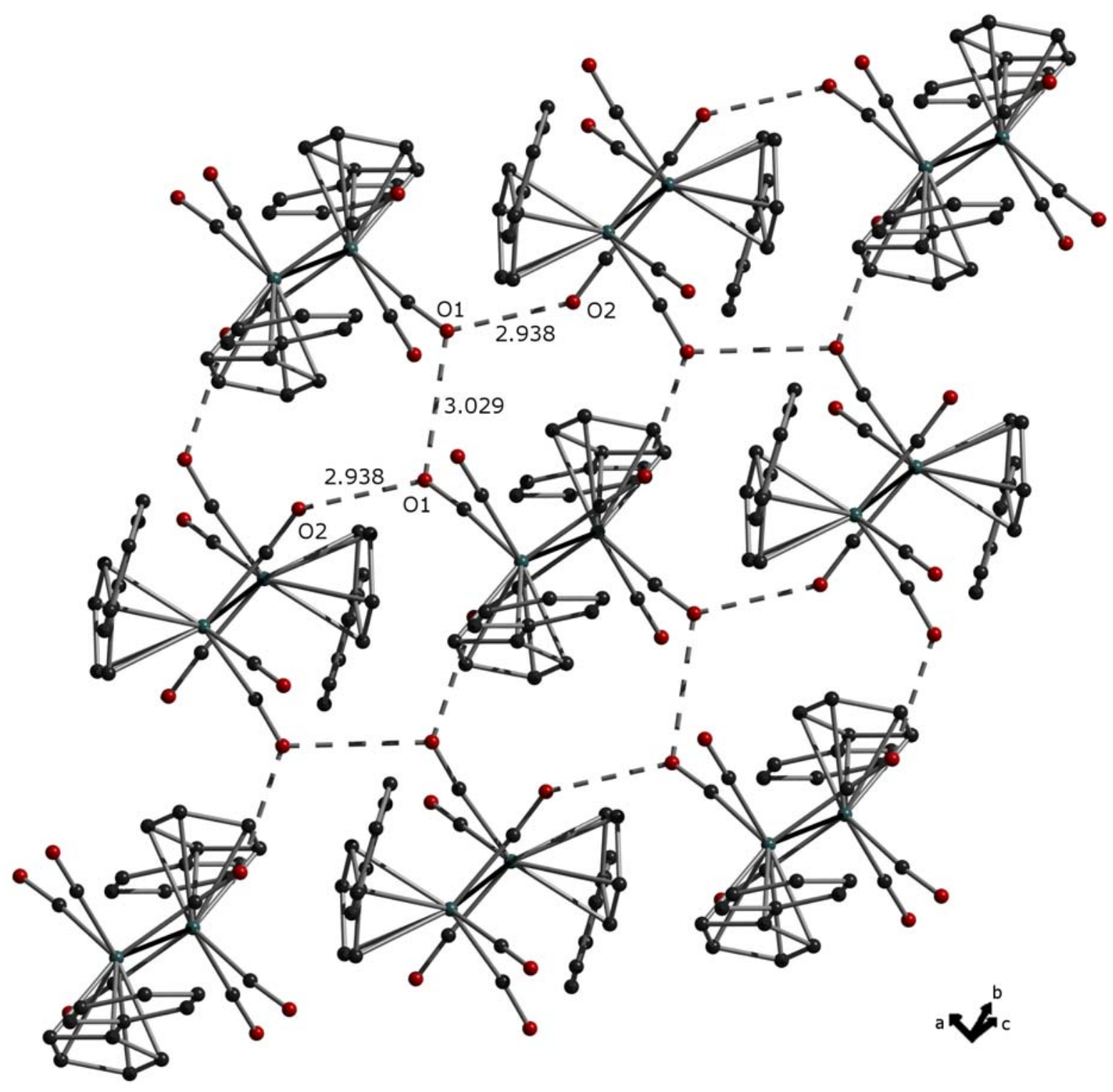

Figure S-1. View of the intermolecular, bifurcated interactions between carbonyl oxygen atoms in $\left[\mathrm{Mo}\left(\eta^{5}-\mathrm{C}_{5} \mathrm{H}_{4} \mathrm{Ph}\right)(\mathrm{CO})_{3}\right]_{2}(\mathbf{1 0})(2.938(4)$ and 3.029(4) $\AA)$ 


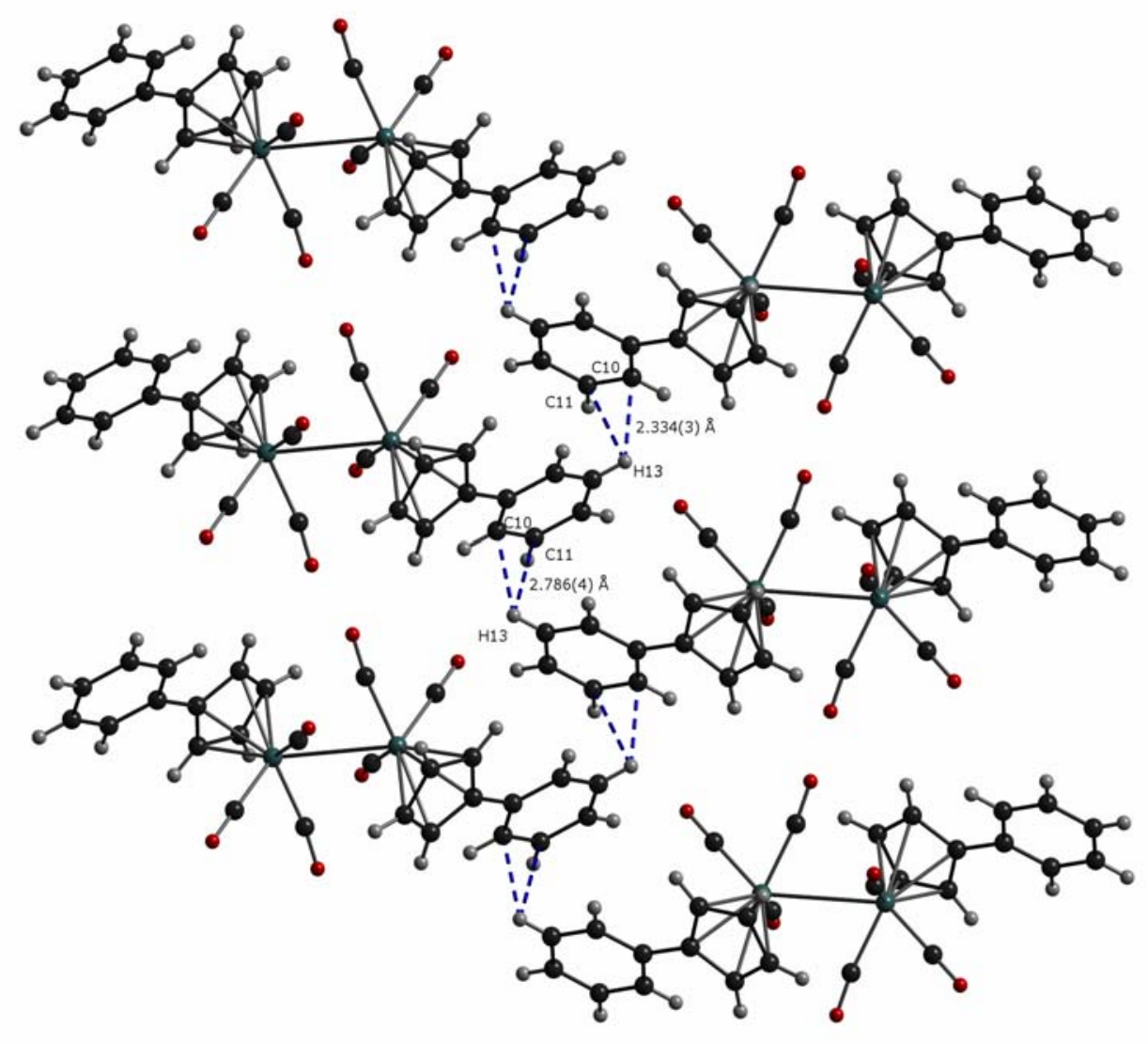

Figure S-2. View of the intermolecular $\mathrm{C}-\mathrm{H} \cdots \pi_{(\mathrm{C}=\mathrm{C})}$ interactions involving the phenyl substituents in $\left[\mathrm{Mo}\left(\eta^{5}-\mathrm{C}_{5} \mathrm{H}_{4} \mathrm{Ph}\right)(\mathrm{CO})_{3}\right]_{2}(\mathbf{1 0})$. 